\title{
Effect of upper-airway stimulation for obstructive sleep apnoea on airway dimensions
}

\author{
Faiza Safiruddin ${ }^{1}$, Olivier M. Vanderveken ${ }^{2,3}$, Nico de Vries ${ }^{1,2,3}$, \\ Joachim T. Maurer ${ }^{4}$, Kent Lee ${ }^{5}$, Quan $\mathrm{Ni}^{5}$ and Kingman P. Strohl ${ }^{6}$ \\ Affiliations: ${ }^{1}$ Sint Lucas Andreas Hospital, Amsterdam, The Netherlands. ${ }^{2}$ Dept of Otorhinolaryngology, Head \\ and Neck Surgery, Antwerp University Hospital, Antwerp, Belgium. ${ }^{3}$ Faculty of Medicine and Health Sciences, \\ University of Antwerp, Antwerp, Belgium. "University Medical Center Mannheim, Mannheim, Germany. \\ ${ }^{5}$ Inspire Medical Systems, Maple Grove, MN, USA. ${ }^{6}$ Division of Pulmonary, Critical Care and Sleep Medicine, \\ Case Medical Center, Case Western Reserve University, Cleveland, OH, USA.
}

Correspondence: Kingman P. Strohl, Division of Pulmonary, Critical Care and Sleep Medicine, Case Medical Center, Case Western Reserve University, 11100 Euclid Ave, Cleveland, OH 44106-1716, USA. E-mail: kpstrohldaol.com

ABSTRACT Upper-airway stimulation (UAS) using a unilateral implantable neurostimulator for the hypoglossal nerve is an effective therapy for obstructive sleep apnoea patients with continuous positive airway pressure intolerance. This study evaluated stimulation effects on retropalatal and retrolingual dimensions during drug-induced sedation compared with wakefulness to assess mechanistic relationships in response to UAS.

Patients with an implanted stimulator underwent nasal video endoscopy while awake and/or during drug-induced sedation in the supine position. The cross-sectional area, anterior-posterior and lateral dimensions of the retropalatal and retrolingual regions were measured during baseline and stimulation.

15 patients underwent endoscopy while awake and 12 underwent drug-induced sedation endoscopy. Increased levels of stimulation were associated with increased area of both the retropalatal and retrolingual regions. During wakefulness, a therapeutic level of stimulation increased the retropalatal area by $56.4 \%$ $(\mathrm{p}=0.002)$ and retrolingual area by $184.1 \%(\mathrm{p}=0.006)$. During stimulation, the retropalatal area enlarged in the anterior-posterior dimension while retrolingual area enlarged in both anterior-posterior and lateral dimensions. During drug-induced sedation endoscopy, the same stimulation increased the retropalatal area by $180.0 \% \quad(p=0.002)$ and retrolingual area by $130.1 \% \quad(p=0.008)$. Therapy responders had larger retropalatal enlargement with stimulation than nonresponders.

UAS increases both the retropalatal and retrolingual areas. This multilevel enlargement may explain reductions of the apnoea-hypopnoea index in selected patients receiving this therapy.

@ERSpublications

Upper-airway stimulation for OSA increases airway dimensions at both the tongue base and the palate http://ow.ly/zZ4hk 


\section{Introduction}

Obstructive sleep apnoea (OSA) is caused by repetitive episodes of complete or partial obstructions of the upper airway during sleep [1]. These repetitive periods of upper airway collapse produce nocturnal hypoxaemia and sleep fragmentation, contributing to a number of OSA-related comorbidities [1]. Continuous positive airway pressure (CPAP) is the first-line treatment for OSA. Despite its efficacy, treatment effectiveness is limited by patient nonadherence, to the point where many with moderate-to-severe disease remain untreated [2]. A number of non-CPAP therapies for moderate-to-severe OSA, such as oral appliance therapy or upper-airway surgery, can be considered as alternatives, but also have their challenges [3-5].

Therapeutic applications using neuromuscular electrical stimulation of the hypoglossal nerve and genioglossus muscle have been designed and evaluated as a potential alternative to positive airway pressure therapy and upper-airway surgical procedures [6-13]. The therapy consists of an implanted, programmable neurostimulation system, with a stimulation electrode around the protrusor branches of the right hypoglossal nerve and a respiration sensor placed in the right intercostal space to detect respiration $[10,14$, 15]. More specifically, upper-airway stimulation (UAS) therapy through unilateral stimulation of the hypoglossal nerve timed with ventilation using an implantable neurostimulator is an effective treatment for selected patients with moderate-to-severe OSA who have failed or are intolerant to CPAP [15].

The mechanism by which UAS prevents collapse deserves further investigation. UAS might be suspected to be of use in patients only with retrolingual collapse; however, empirically, it appears that stimulation may alleviate apnoeas that also have a component of retropalatal collapse [16, 17]. While the effect of UAS on retrolingual area can be directly attributed to tongue-base advancement through activation of the genioglossus muscle, the effect of stimulation on retropalatal area is less intuitive. During acute hypoglossal nerve stimulation, other groups have demonstrated increased retrolingual and retropalatal lucency in sagittal neck fluoroscopic images during periods of acute stimulation during surgery, indicating an increase of airway size in the anterior-posterior dimension [16]; however, this two-dimensional approach using a single stimulation setting may not fully capture quantitative and dynamic upper-airway dimensions. Observations during graded stimulation would better describe the action and help develop an understanding of UAS effects.

We used awake nasopharyngoscopic evaluation [18] and drug-induced sedation endoscopy (DISE). The latter technique is a widely used method to determine the degree, configuration and site of upper-airway obstruction when selecting patients for non-CPAP treatments for OSA [19]. DISE offers a method to visually quantify upper-airway changes during sedation, which has been shown to simulate upper-airway collapse during sleep $[20,21]$. Such a controlled setting permits evaluation of differing stimulation levels.

In this study, we measured the effects of graded stimulation on multilevel airway dimensions during wakefulness and DISE in OSA patients with chronically implanted UAS systems. The hypotheses were that stimulation would simultaneously enlarge retropalatal and retrolingual airway dimensions compared with no stimulation, and that increasing stimulation amplitude would produce larger upper-airway dimensions compared with periods of no stimulation.

\section{Methods}

\section{Patient selection}

A subset of patients were enrolled from three European centres participating in a multicentre, prospective trial for the safety and efficacy of an implantable UAS system (Inspire Medical Systems, Minneapolis, MN, USA) for the treatment of moderate-to-severe OSA in patients intolerant to CPAP [15]. Per protocol, all patients underwent DISE screening prior to implant, and patients with retropalatal complete concentric collapse were excluded from implantation. Other pre-surgical selection criteria have been described separately [15]. All patients had provided written informed consent for the study, and the protocol was approved by the local medical ethics committees.

2 and 6 months after implantation, patients had overnight titration polysomnography (PSG) to identify the therapeutic stimulation amplitude needed to abolish respiratory events during sleep, and 12 months after implant, a PSG was performed at the identified therapeutic amplitude. At least 2 months after implantation, patients from three European centres in this study were invited to participate in an awake endoscopy and/or DISE.

\section{Awake endoscopy}

Awake nasal endoscopy was performed with the patient in the supine position, during nasal breathing. The endoscope was inserted through the nasal cavity until the retropalatal region was visualised. Stimulation was applied at four increasing amplitudes, representing first sensation, bulk tongue movement, 
therapeutic level determined from a titration sleep study and sub-discomfort, while ensuring the endoscope position remained unchanged during periods with and without stimulation. This same technique was performed after distal positioning to observe the retrolingual level.

\section{Drug-induced sedation endoscopy}

After awake endoscopy, with the patient still in the supine position, propofol and/or midazolam were administered as sedatives [19]. DISE was performed in the same manner as the pre-implant screening, following the study's DISE protocol. The targeted sedation depth was a loss of response to verbal stimulation and the presence of snoring and/or obstructed airway events [19]. When the sedation target was achieved, the endoscope was inserted distally to visualise the nasopharyngeal, retropalatal, oropharyngeal, retrolingual and retroepiglottic regions, and assess the baseline direction and degree of upper-airway collapse over several respiratory cycles during inspiration using a standardised protocol [15]. Stimulation was applied at the same amplitudes during DISE as during wakefulness, and endoscopic images of the retropalatal and retrolingual regions were captured in the same manner.

\section{Image analysis}

Images were captured prior to stimulation and during stimulation in both the retropalatal and retrolingual region. Images where the retropalatal or retrolingual region could not be visualised due to saliva, air bubbles, lens fogging or other visual artefacts were excluded from the analysis. Images of the retropalatal and retrolingual regions from the periods just prior to and during UAS were digitally measured by a single observer (F. Safiruddin) using ImageJ (US National Institutes of Health, Bethesda, MD, USA).

Quantitative measurement of upper-airway dimensions was performed similarly to the method as described by BOREK et al. [20]. During baseline and stimulation, the anterior-posterior and lateral dimensions of the airway were measured at the centre of the airway lumen. Cross-sectional area and dimensions were calculated as a percentage of the total scope image to normalise for the varying digital image resolution of endoscopy machines among investigational centres.

\section{Comparison of upper-airway area with the 12-month apnoea-hypopnoea index response criterion} Analysis of the endoscopic images was combined with the apnoea-hypopnoea index (AHI) from the 12-month visit to understand the association between changes in upper-airway area during stimulation and the change in AHI between responders and nonresponders. Therapy response was prospectively defined as a $50 \%$ reduction of the AHI from baseline to $<20$ events $\cdot \mathrm{h}^{-1}[22,23]$.

\section{Statistical analysis}

Descriptive statistics were calculated for demographic variables and upper-airway area measurements. Wilcoxon signed rank tests were used to compare measurements between periods before and during stimulation, and the Mann-Whitney-Wilcoxon two-sample test was used for comparisons between responders and nonresponders. A p-value $<0.05$ was considered statistically significant.

\section{Results}

\section{Patient demographics}

15 patients underwent awake nasal endoscopy; 12 patients underwent DISE. One patient was excluded from awake endoscopy due to gag reflex caused by the endoscope, thus leaving 11 patients with both measurements. Demographic and baseline information are presented in table 1.

TABLE 1 Description of the cohort undergoing endoscopic evaluation

\begin{tabular}{lcc} 
& Awake endoscopy & $\begin{array}{c}\text { Drug-induced } \\
\text { sedation endoscopy }\end{array}$ \\
\hline Subjects & 15 & 12 \\
Age years & $50.4 \pm 10.2$ & $51.2 \pm 9.0$ \\
Males \% & 100 & 100 \\
Body mass index $\mathbf{k g} \cdot \mathbf{m}^{-\mathbf{2}}$ & $27.9 \pm 1.9$ & $28.2 \pm 1.5$ \\
Baseline apnoea-hypopnoea index events $\mathbf{h}^{-\mathbf{1}}$ & $29.3 \pm 7.5$ & $28.8 \pm 7.8$ \\
Responders/nonresponders at $\mathbf{1 2}$ months & $8 / 7$ & $6 / 6$ \\
\hline
\end{tabular}

Data are presented as $\mathrm{n}$ or mean $\pm \mathrm{SD}$, unless otherwise stated. 


\section{Awake airway dimensions during stimulation}

Both retropalatal and retrolingual regions were open without stimulation, when patients were breathing normally through the nose. With stimulation, mild tongue protrusion was visible. From an endoscopic view of the upper airway, there was an immediate increase in retropalatal and retrolingual area. Airway area returned to baseline after stimulation and the tongue returned to its resting position (fig. 1). Increasing stimulation amplitudes were associated with progressively larger area (fig. 2).

During awake stimulation at the therapeutic amplitude, retropalatal area increased by $56.4 \%$ and retrolingual area increased by $184.1 \%$, as compared with the area without stimulation ( $\mathrm{p}=0.002$ and $\mathrm{p}=0.006$, respectively). The retrolingual area appeared the most sensitive to stimulation, as the lowest stimulation amplitude (sensation) was sufficient to increase retrolingual area compared with no stimulation $(\mathrm{p}<0.005)$ but a higher stimulation amplitude (bulk movement) was required to significantly increase retropalatal area versus no stimulation $(\mathrm{p}=0.002)$.

In regard to shape, stimulation increased the retropalatal anterior-posterior dimension from baseline without changes in the laterolateral dimension, while stimulation increased retrolingual in both anteriorposterior and laterolateral dimensions as compared to baseline (table 2).

\section{Sedated airway dimensions during stimulation}

Sedation decreased cross-sectional area at both the retropalatal and retrolingual levels, compared with awake endoscopy. At baseline without stimulation, the majority of patients had retropalatal and retrolingual airway collapse $(n=9)$, while three patients had only retrolingual collapse. Anterior-posterior

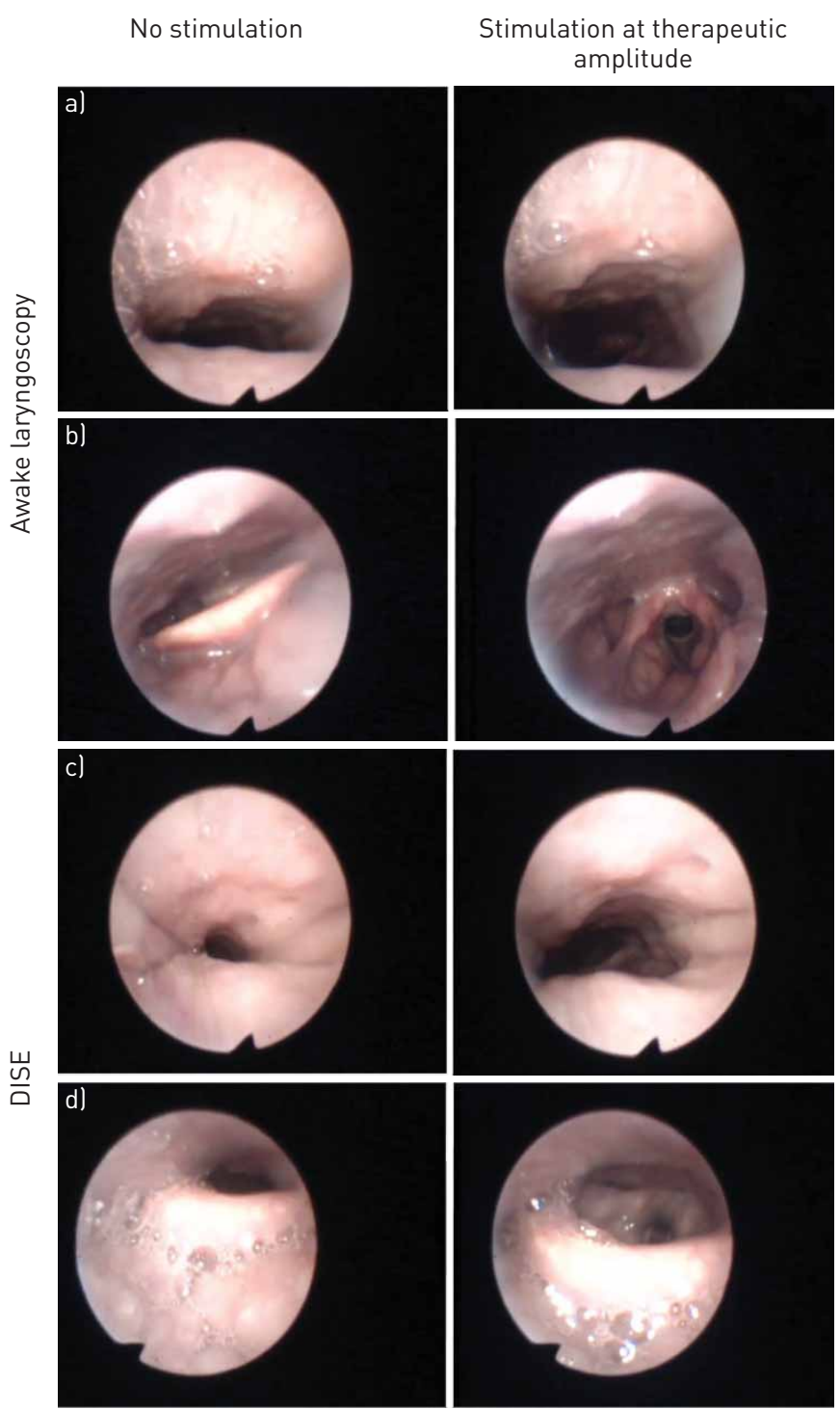

FIGURE 1 Example of airway images from the same patient during $a, b$ ) awake endoscopy and $\mathrm{c}$, d) drug-induced sedation endoscopy (DISE). a, c) At the top of the images is the posterior pharyngeal wall and at the bottom is the soft palate. b, d) At the top of the images is the posterior wall and at the bottom is the tongue base. 



FIGURE 2 a) Retropalatal and b) retrolingual area before and during stimulation at the four functional stimulation amplitudes while awake. Bars represent the mean and whiskers the standard deviation.

collapse was the most common collapse direction in the retropalatal region. At the retropalatal level, four patients had complete anterior-posterior collapse, three patients had partial anterior-posterior collapse and five patients had no palatal collapse. At the retrolingual level, nine patients had complete anteriorposterior collapse, two patients had partial anterior-posterior collapse and one patient had complete concentric collapse.

Similar to awake endoscopy, stimulation at the bulk-movement amplitude and higher amplitudes led to significantly increased retropalatal and retrolingual area compared with no stimulation (fig. 3). During stimulation at the therapeutic amplitude, retropalatal area increased by $180.0 \%(\mathrm{p}=0.002)$ and retrolingual area increased by $130.1 \%(\mathrm{p}=0.008)$. There was a progressive increase in retropalatal and retrolingual area with higher stimulation levels compared with no stimulation, although increases in retrolingual area appeared to plateau beyond the bulk movement threshold (fig. 4).

The increase in airway area with stimulation at the therapeutic amplitude during DISE was due to statistically significant anterior-posterior enlargement at the retropalatal region without any change in laterolateral length. There was anterior-posterior and laterolateral enlargement at the retrolingual region, although the change in laterolateral dimension was slightly short of meeting statistical significance (table 2).

\section{Comparing relative changes in upper-airway area between awake endoscopy and DISE}

The percentage change in area between periods without and with stimulation was used to compare the effect of stimulation amplitudes on open-airway area between awake endoscopy and DISE. The percentage change was measured instead of an absolute difference to control for any variation in endoscope positional depth between awake endoscopy and DISE.

Stimulation during awake endoscopy had a similar percentage increase in retrolingual and retropalatal area. During DISE, however, stimulation had a larger percentage increase in retropalatal than retrolingual area. This was due to the greater baseline collapse in retropalatal area during DISE when compared with

TABLE 2 Effect of stimulation on retropalatal and retrolingual area, and dimensions during awake endoscopy and drug-induced sedation endoscopy, reported as a percentage of the endoscope image size

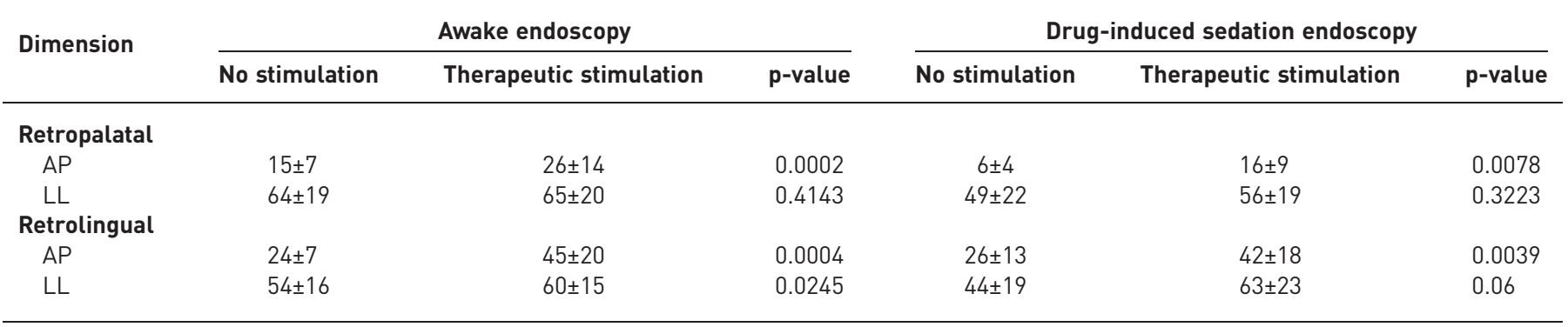

Data are presented as mean \pm SD, unless otherwise stated. AP: anterior-posterior; LL: laterolateral. 


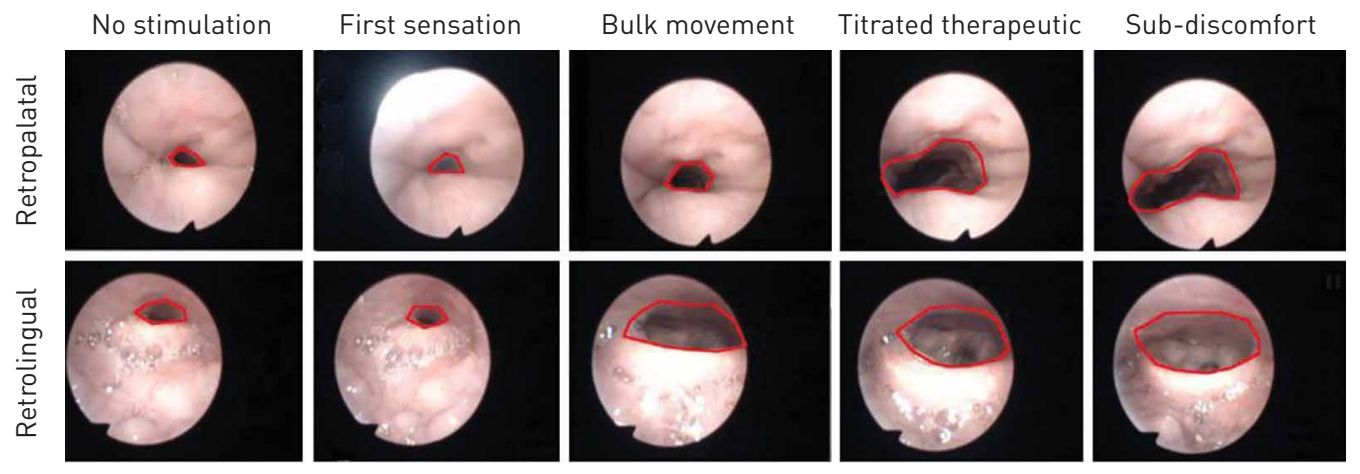

FIGURE 3 Increases in retropalatal and retrolingual area comparing no stimulation with progressively higher levels of stimulation during drug-induced sedation endoscopy. The outline indicates the measured retropalatal and retrolingual area; this area was recorded as a percentage of the total endoscope image area. Retropalatal images (top) are orientated with the posterior pharyngeal wall at the top of the image and the soft palate/uvula at the bottom of the image. Retrolingual images (bottom) are similarly orientated, with the posterior tongue base and epiglottis at the bottom of the image.

the retrolingual level. Proportionately, changes in retropalatal area were nearly three times more expansive to stimulation during DISE than awake, whereas the retrolingual region was 1.3 times more expansive to stimulation during DISE than awake endoscopy (fig. 5).

\section{Comparison of stimulation effects on airway area in responders and nonresponders}

Stimulation reduced the AHI in responders from $29 \pm 3$ events $\cdot \mathrm{h}^{-1}$ at baseline to $8 \pm 3$ events $\cdot \mathrm{h}^{-1}$ at 12 months $(\mathrm{p}=0.02)$, while nonresponders had no change in AHI $\left(31 \pm 10\right.$ events $\cdot \mathrm{h}^{-1}$ to $43 \pm 23$ events $\left.\cdot \mathrm{h}^{-1}, \mathrm{p}=0.30\right)$. Using the awake endoscopy data, both responders and nonresponders had a statistically significant increase in retrolingual area during stimulation at the therapeutic amplitude, compared with no stimulation. However, responders had a statistically significant increase in retropalatal area during stimulation, while in contrast, nonresponders had a smaller increase in retropalatal area during stimulation, which was not statistically significant. There was no difference in the degree of retrolingual area change during stimulation between responders and nonresponders $(\mathrm{p}=0.71)$; however, responders had a greater degree of retropalatal enlargement during stimulation than nonresponders $(\mathrm{p}=0.047)$ (table 3$)$.

\section{Discussion}

The key findings in this study confirm the hypotheses that unilateral stimulation of the hypoglossal nerve using an implantable UAS system, timed with ventilation, increases airway area at multiple levels and that the degree of upper-airway opening corresponds to higher amplitudes of stimulation. These findings of multilevel opening at the different collapsible levels of the upper airway with stimulation probably explain the effectiveness of UAS therapy in reducing AHI severity in a recent multicentre, prospective trial evaluating this UAS therapy, as well as explain the return to baseline AHI in patients who were randomised to therapy withdrawal [15].
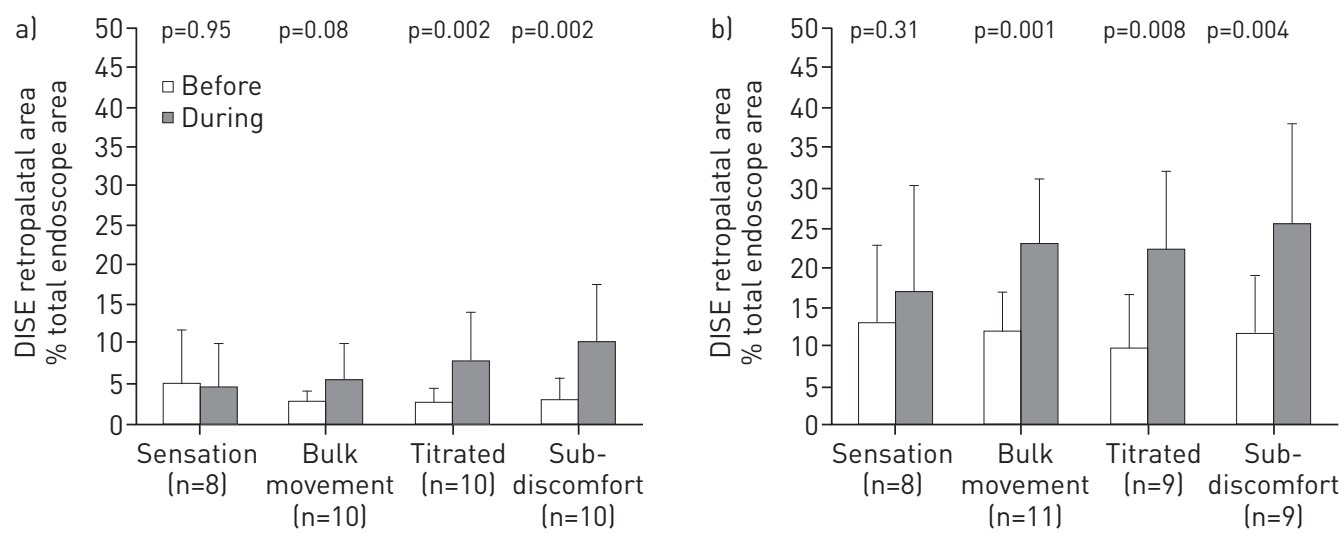

FIGURE 4 a) Retropalatal and b) retrolingual airway area before and during stimulation, while undergoing drug-induced sedation endoscopy (DISE). Bars represent the mean and whiskers the standard deviation. 



FIGURE 5 Comparing the effect of stimulation on a) retropalatal and b) retrolingual airway area between awake endoscopy and drug-induced sedation endoscopy (DISE). p-values are for comparisons with the area at the sensation amplitude.

The findings of improvement in upper-airway cross-sectional area at the retropalatal level during unilateral hypoglossal nerve stimulation are in line with the results from groups visualising retropalatal area during direct genioglossus muscle stimulation under anaesthesia $[6,8]$, as well as others visualising palatal anterior displacement and thinning from fluoroscopic images during intraoperative testing of an implantable hypoglossal neurostimulator [16]. The present study found this result in patients undergoing a therapeutic clinical trial.

The effect of stimulation on anterior-posterior and lateral dimensions of the airway is relevant, particularly regarding the role of airway shape for OSA therapies. In general, a retropalatal shape change from circular to elliptical, specifically a lateral elliptical shape, is believed to be a therapy mechanism of CPAP [24] and oral appliances [18]. In this study, the dominant effect of stimulation was the enlargement of the retropalatal and retrolingual airway in the anterior-posterior dimension, which may be due to patient selection, the therapy mechanism, or both. First, patients were pre-selected with retropalatal anterior-posterior collapse, and patients with retropalatal concentric collapse were excluded because of data indicating they would benefit less from the therapy [17]. The stimulation lead was placed to recruit the genioglossus muscle, resulting in an anterior-posterior enlargement of at least the retro-lingual airway area during stimulation. Activation of the genioglossus may address a particular phenotype of OSA with blunted genioglossus activity [25]. In addition to the increased anterior-posterior dimension, there was also an additive retrolingual lateral effect, albeit a smaller one. The finding of an increased anteriorposterior retropalatal dimension, and an increased anterior-posterior and lateral retrolingual dimension from stimulation may suggest an additive therapy mechanism that differs from that of oral appliances, which were associated with increases in lateral retropalatal dimension and anterior-posterior retrolingual dimension [18].

Resolving multilevel collapse occurring at the palate and tongue base is probably crucial in order to achieve treatment success. While a majority of patients with an established diagnosis of moderate-to-severe OSA have multilevel collapse, as also seen in this cohort, the soft palate is the most collapsible region of the upper airway [26]. In this study, the palate demonstrated the largest improvement in terms of increase in cross-sectional area at that specific upper-airway level during stimulation, in part due to reduced baseline area before stimulation during DISE. When comparing responders with nonresponders, the degree of retropalatal enlargement in response to stimulation was statistically significant only in the responders.

The mechanisms by which hypoglossal stimulation increases retropalatal area deserve consideration. Retrolingual opening is expected from stimulation of the genioglossus, but the that of the retropalatal region is not. One concept is that the retropalatal effect seen during stimulation is due to a mechanical linkage between the soft palate and the tongue base $[27,28]$. Anatomically, the soft palate is linked to the tongue base through the anterior palatal pillar. The anterior pillar also contains the palatoglossus muscle, which courses through the soft palate and the uvula, and inserts into the sides of the tongue [29]. This structure can have a passive and active effect of pulling the soft palate inferiorly and anteriorly. The palatoglossus muscle exhibits phasic respiratory electromyographic activity and has a reflex activation to negative pressure in OSA patients [29], which suggests it could play a role in restoring airway patency. When stimulation moves the tongue base forward, it might result in palatal advancement through anterior 
TABLE 3 Comparing the effect of stimulation on retropalatal and retrolingual area in responders and nonresponders, reported as a percentage of the endoscope image size

\begin{tabular}{|c|c|c|c|c|c|c|c|}
\hline & \multirow{2}{*}{ Subjects $\mathbf{n}$} & \multicolumn{3}{|c|}{ Retropalatal area } & \multicolumn{3}{|c|}{ Retrolingual area } \\
\hline & & No stimulation & Therapeutic stimulation & p-value & No stimulation & Therapeutic stimulation & p-value \\
\hline Responders & 7 & $11 \pm 10$ & $22 \pm 21$ & 0.031 & $10 \pm 5$ & $26 \pm 20$ & 0.016 \\
\hline
\end{tabular}

Data are presented as mean $\pm \mathrm{SD}$, unless otherwise stated.

displacement of the anterior palatal pillar and the palatoglossus muscle, moving the soft palate anteriorly, and causing an increase in the retropalatal cross-sectional area and dimensions.

A passive effect of tongue-base manipulation causing retropalatal opening can be inferred from other studies. In an animal model, forward motion of the hyoid apparatus caused nasopharynx opening [28]. Furthermore, mandibular advancement devices, which also have the effect of moving the tongue base due to its attachment to the mandible, have demonstrated an increase in retropalatal area to varying degrees $[30,31]$. Furthermore, computed tomographic imaging of the airway while wearing a mandibular device demonstrated increases in retropalatal and retrolingual airway area [32].

Variation in the retropalatal area response to stimulation among patients was observed. Responders and nonresponders had similar degrees of retrolingual opening to stimulation; however, responders had a greater increase in retropalatal area. One potential explanation for these differences is that the linkages between retropalatal and retrolingual regions may vary among individuals. This interconnection between hypoglossal activation and upper-airway structural movement deserves further investigation.

This study is consistent with previous work that increasing stimulation amplitudes increases airflow, until reaching a plateau at higher amplitudes [13]. The previously reported plateau effect in flow is probably explained by the plateau of airway size at the retrolingual area, as seen in this study. There may be two reasons for this ceiling effect. First, the passive distension of the pharynx is limited by surrounding tissue boundaries such as the hard palate or the spine. Second, if all fibres of the genioglossus muscle are activated, there is a diminishing effect of higher amplitudes, as further increase in stimulation is less likely to further stabilise the upper airway. The relationship of amplitude with increases of airway area and airflow is relevant to the clinical management of OSA patients with UAS. It suggests that the stimulation amplitude can be titrated to a level sufficient to abolish OSA events, similar to CPAP, yet below a level that would arouse the patient from sleep. In our study, a significant increase in airway area was obtained at stimulation amplitudes less than the amplitude that would be uncomfortable while awake. Thus, UAS can be effectively titrated during natural sleep in the clinical setting without arousing the patient.

\section{Limitations}

The patients in this analysis were a subset of the patients reported in a separate investigation evaluating the efficacy of stimulation on the AHI on a larger cohort. It should be noted that the baseline age, body mass index and AHI of the patients in this subanalysis were similar to the entire cohort in the larger trial [15]. Nevertheless, as this investigation was a subgroup analysis of patients in a larger clinical trial, the conclusions of this paper may not be generalisable to the larger cohort. Furthermore, the comparison of airway responses to stimulation between responders and nonresponders was possible by combining the endoscopic results of this investigation, which preceded the polysomnographic data from the 12-month post-implant visit. Our findings regarding the upper-airway responses to stimulation in responders and nonresponders are preliminary and in a limited number; however, this suggests the differential response to stimulation at the retropalatal airway deserve further investigation.

Due to protocol design, patients were pre-selected for implantation to be without retropalatal complete concentric collapse, and had various degrees of retropalatal anterior-posterior collapse [15]. Thus, our findings are limited to this study population. We made the decision to use stimulation amplitude according to functional action rather than fixed numeric amplitudes. Due to individual differences in electrical conductance, nerve-electrode impedances and individualised stimulation parameters among patients, we chose to compare functional stimulation amplitudes instead of absolute stimulation amplitudes. Furthermore, the individual stimulation amplitudes at sensation threshold, bulk tongue movement, therapeutic amplitude for sleep and sub-discomfort level describe comparable functional responses to stimulation across subjects. Therefore, we believe the choice of amplitude setting in this manner is reasonable. 
Regarding the reliability of DISE compared to natural sleep, a study of respiratory events in non-OSA controls and OSA patients undergoing PSG during propofol sedation and diurnal sleep did not find significant differences in apnoea severity [33]; thus, to some extent, DISE may be an approximation of upper-airway function with sedation. Variability during DISE assessment may occur due to differences in sedation depth during the procedure or between patients, which may influence collapsibility. In our study, the DISE procedure was set by protocol, with a sedation depth target of loss of verbal response to stimuli, and the presence of snoring and/or sleep apnoea events [19]. While our approach was based on visual symptoms of airway obstruction, quantitative methods for measuring DISE sedation depth via electroencephalography have also been suggested [34]. Other DISE limitations include a time-dependent effect of sedation on upper-airway collapse and/or shape, after the initiation of a stable moderate sedation plane. Confounding by subtle changes in sedation state could be avoided in the future by applying the stimulation amplitudes in random order.

The use of nasopharyngoscopic endoscopy is dependent on image quality and visibility of the upper-airway structures. The multicentre nature of this study led to use of different endoscopy systems with different imaging resolutions, making it difficult to compare images among centres directly. Furthermore, the exact position of the endoscope camera tip in the airway was not standardised between awake endoscopy and DISE, making it difficult to compare images among patients, and between awake and sedation endoscopy in the same patient. To address this issue, we normalised our measurements to the total endoscope image, which was unchanged between periods with and without stimulation. Future studies would use an endoscope with calibrated distance markers to determine precise scope depth [20]. Another limitation is the lack of information on airway stiffness during stimulation, as changes in airway stiffness, particularly along the longitudinal dimension of the airway, may not be visible when measuring changes in area or dimensions during DISE or awake laryngoscopy. Concerning DISE, the impact of interand intra-observer variations has been evaluated in different studies that indicate that variability is moderate to substantial $[21,35]$.

\section{Conclusion}

UAS of the hypoglossal nerve increases retropalatal and retrolingual area, primarily in the anteroposterior direction. The mechanism of retropalatal opening with stimulation is reasoned to be due to a linkage to the tongue-base effects, while retrolingual opening happens due to unilateral hypoglossal stimulation. The reductions of AHI in patients with UAS are probably due to a multilevel effect on upper-airway opening during hypoglossal nerve stimulation timed with ventilation.

\section{References}

1 Dempsey JA, Veasey SC, Morgan BJ, et al. Pathophysiology of sleep apnea. Physiol Rev 2010; 90: 47-112.

2 Stuck BA, Leitzbach S, Maurer JT. Effects of continuous positive airway pressure on apnea-hypopnea index in obstructive sleep apnea based on long-term compliance. Sleep Breath 2012; 16: 467-471.

3 Vanderveken OM, Devolder A, Marklund M, et al. Comparison of a custom-made and a thermoplastic oral appliance for the treatment of mild sleep apnea. Am J Respir Crit Care Med 2008; 178: 197-202.

4 Weaver TE, Grunstein RR. Adherence to continuous positive airway pressure therapy: the challenge to effective treatment. Proc Am Thorac Soc 2008; 5: 173-178.

5 Weaver EM. Sleep apnea devices and sleep apnea surgery should be compared on effectiveness, not efficacy. Chest 2003; 123: 961-962.

6 Dotan Y, Golibroda T, Oliven R, et al. Parameters affecting pharyngeal response to genioglossus stimulation in sleep apnoea. Eur Respir J 2011; 38: 338-347.

7 Kezirian EJ, Boudewyns A, Eisele DW, et al. Electrical stimulation of the hypoglossal nerve in the treatment of obstructive sleep apnea. Sleep Med Rev 2010; 14: 299-305.

8 Oliven A, Tov N, Geitini L, et al. Effect of genioglossus contraction on pharyngeal lumen and airflow in sleep apnoea patients. Eur Respir J 2007; 30: 748-758.

9 Schwartz AR, Bennett ML, Smith PL, et al. Therapeutic electrical stimulation of the hypoglossal nerve in obstructive sleep apnea. Arch Otolaryngol Head Neck Surg 2001; 127: 1216-1223.

10 Van de Heyning PH, Badr MS, Baskin JZ, et al. Implanted upper airway stimulation device for obstructive sleep apnea. Laryngoscope 2012; 122: 1626-1633.

11 Eastwood PR, Barnes M, Walsh JH, et al. Treating obstructive sleep apnea with hypoglossal nerve stimulation. Sleep 2011; 34: 1479-1486.

12 Mwenge GB, Rombaux P, Dury M, et al. Targeted hypoglossal neurostimulation for obstructive sleep apnoea: a 1-year pilot study. Eur Respir J 2013; 41: 360-367.

13 Schwartz AR, Barnes M, Hillman D, et al. Acute upper airway responses to hypoglossal nerve stimulation during sleep in obstructive sleep apnea. Am J Respir Crit Care Med 2012; 185: 420-426.

14 Maurer JT, Van de Heyning P, Lin HS, et al. Operative technique of upper airway stimulation: an implantable treatment of obstructive sleep apnea. Oper Techn Otolaryngol 2012; 23: 227-233.

15 Strollo PJ Jr, Soose RJ, Maurer JT, et al. Upper-airway stimulation for obstructive sleep apnea. N Engl J Med 2014; 370: 139-149.

16 Goding GS Jr, Tesfayesus W, Kezirian EJ. Hypoglossal nerve stimulation and airway changes under fluoroscopy. Otolaryngol Head Neck Surg 2012; 146: 1017-1022. 
17 Vanderveken OM, Maurer JT, Hohenhorst W, et al. Evaluation of drug-induced sleep endoscopy as a patient selection tool for implanted upper airway stimulation for obstructive sleep apnea. J Clin Sleep Med 2013; 9: 433-438.

18 Chan AS, Lee RW, Srinivasan VK, et al. Nasopharyngoscopic evaluation of oral appliance therapy for obstructive sleep apnoea. Eur Respir J 2010; 35: 836-842.

19 De Vito A, Carrasco Llatas M, Vanni A, et al. European position paper on drug-induced sedation endoscopy (DISE). Sleep Breath 2014; 18: 453-465.

20 Borek RC, Thaler ER, Kim C, et al. Quantitative airway analysis during drug-induced sleep endoscopy for evaluation of sleep apnea. Laryngoscope 2012; 122: 2592-2599.

21 Vroegop AV, Vanderveken OM, Dieltjens M, et al. Sleep endoscopy with simulation bite for prediction of oral appliance treatment outcome. J Sleep Res 2013; 22: 348-355.

22 Lin HC, Friedman M, Chang HW, et al. The efficacy of multilevel surgery of the upper airway in adults with obstructive sleep apnea/hypopnea syndrome. Laryngoscope 2008; 118: 902-908.

23 Sher AE, Schechtman KB, Piccirillo JF. The efficacy of surgical modifications of the upper airway in adults with obstructive sleep apnea syndrome. Sleep 1996; 19: 156-177.

24 Leiter JC. Upper airway shape: is it important in the pathogenesis of obstructive sleep apnea? Am J Respir Crit Care Med 1996; 153: 894-898.

25 Eckert DJ, White DP, Jordan AS, et al. Defining phenotypic causes of obstructive sleep apnea. Identification of novel therapeutic targets. Am J Respir Crit Care Med 2013; 188: 996-1004.

26 Trudo FJ, Gefter WB, Welch KC, et al. State-related changes in upper airway caliber and surrounding soft-tissue structures in normal subjects. Am J Respir Crit Care Med 1998; 158: 1259-1270.

27 Kuehn DP, Azzam NA. Anatomical characteristics of palatoglossus and the anterior faucial pillar. Cleft Palate J 1978; 15: 349-359.

28 Mortimore IL, Douglas NJ. Palatal muscle EMG response to negative pressure in awake sleep apneic and control subjects. Am J Respir Crit Care Med 1997; 156: 867-873.

29 Van de Graaff WB, Gottfried SB, Mitra J, et al. Respiratory function of hyoid muscles and hyoid arch. $J$ Appl Physiol Respir Environ Exerc Physiol 1984; 57: 197-204.

30 Chan AS, Sutherland K, Schwab RJ, et al. The effect of mandibular advancement on upper airway structure in obstructive sleep apnoea. Thorax 2010; 65: 726-732.

31 Isono S, Tanaka A, Sho Y, et al. Advancement of the mandible improves velopharyngeal airway patency. J Appl Physiol (1985) 1995; 79: 2132-2138.

32 Choi JK, Hur YK, Lee JM, et al. Effects of mandibular advancement on upper airway dimension and collapsibility in patients with obstructive sleep apnea using dynamic upper airway imaging during sleep. Oral Surg Oral Med Oral Pathol Oral Radiol Endod 2010; 109: 712-719.

33 Rabelo FA, Braga A, Kupper DS, et al. Propofol-induced sleep: polysomnographic evaluation of patients with obstructive sleep apnea and controls. Otolaryngol Head Neck Surg 2010; 142: 218-224.

34 Hillman DR, Platt PR, Eastwood PR. Anesthesia, sleep, and upper airway collapsibility. Anesthesiol Clin 2010; 28 : 443-455.

35 Kezirian EJ, White DP, Malhotra A, et al. Interrater reliability of drug-induced sleep endoscopy. Arch Otolaryngol Head Neck Surg 2010; 136: 393-397. 